\title{
Photo-Induced Formation of Peroxide in Saccharides and Related Compounds
}

\author{
Hitoshi Kubota, ${ }^{*}$ Yoshitaka Ogiwara, ${ }^{*}$ and Kei Matsuzaki** \\ *Department of Polymer Chemistry, Faculty of Engineering, \\ Gunma University, Kiryu, Gunma 376, Japan. \\ **Department of Industrial Chemistry, Faculty of Engineering, \\ University of Tokyo, Bunkyo-ku, Tokyo 113, Japan.
}

(Received May 17, 1976)

\begin{abstract}
Photo-induced formations of peroxide in saccharides, poly(vinyl alcohol) (PVA), and cellulose were examined in aqueous media, and the reaction mechanisms were discussed. Three factors, light shorter than $300 \mathrm{~nm}$, saccharide, and oxygen, were essential to the formation of peroxide. Greater amounts of peroxide were formed with reducing saccharides, D-glucose, D-fructose, and cellobiose than with nonreducing saccharides, sucrose and methyl- $\alpha$-D-glucopyranoside. Based on the determinations of reducing power and carbonyl group, spectrophotometric measurements, and the detection of lactone in photo-irradiated aqueous solutions of D-glucose, reaction mechanisms involving the formation of hydrogen peroxide and the change of D-glucose into lactone or ketone structure were discussed. The formation of peroxide was also observed in PVA and cellulose samples. The peroxide was postulated to be hydrogen peroxide. It is concluded that the structure - $\mathrm{HC}(\mathrm{OH})$ - of saccharide and related compounds contributes generally to the formation of hydrogen peroxide induced by a photolysis reaction.

KEY WORDS Reducing Saccharide / Cellulose / Poly(vinyl alcohol) / UV Irradiation / Photooxidation / Dehydrogenation / UV Spectrum / Reducing Power / Hydrogen Peroxide /
\end{abstract}

In a previous paper, ${ }^{1}$ the authors observed that the photopolymerization of methyl methacrylate in aqueous medium in an irradiated system with light longer than $300 \mathrm{~nm}$ was accelerated by addition of aldose and ketose, Dfructose especially showing a marked promoting action. Moreover, aqueous solutions of D-fructose preirradiated in air atmosphere with ultraviolet light (UV) shorter than $300 \mathrm{~nm}$ activated the photopolymerization of methyl methacrylate, leading to an increased conversion with the time of preirradiation. This phenomenon is inferred to be the contribution of peroxide in the preirradiated aqueous solution of $\mathrm{D}$-fructose to the initiation of polymerization. In fact, the formation of peroxide was confirmed in the irradiated aqueous solution of saccharide and the amount was determined iodometrically.

Phillips $^{2}$ reviewed studies on the photolysis of mono- to polysaccharides, in which changes in optical rotation and reducing property, scission of glycosidic bonds, formation of acids, and liberation of carbon monoxide, carbon dioxide, and hydrogen were considered to be characteristic of the photolysis of saccharides. In the present paper, peroxide formed in various UV-irradiated aqueous solutions of saccharides was examined, and the mechanism of peroxide formation in the photolysis of saccharide was discussed. Then, investigations in the same way on poly(vinylalcohol) (PVA) and cellulose, compounds related to saccharides, were carried out to confirm the reaction mechanism.

\section{EXPERIMENTAL}

The various saccharides used were of reagent grade in commercial origin. Somogyi's method described in the previous paper ${ }^{1}$ was employed 
in the determination of the reducing power of the saccharides. Peroxide was determined according to iodometry. Namely, $2 \mathrm{ml}$ of $5-N$ sulfuric acid and $5 \mathrm{ml}$ of $20-\%$ aqueous solution of potassium iodide were added to $10 \mathrm{~m} l$ of irradiated aqueous solution of saccharide. After standing for $20 \mathrm{~min}$ in a dark place, the liberated iodine was titrated with $0.003-N$ sodium thiosulfate with starch as indicator. The same titration was carried out on unirradiated aqueous solution of the saccharide as blank test. With the use of a Toshiba high-pressure mercury lamp H400-P $(400 \mathrm{~W})$ the irradiations were conducted in a quartz glass tube about $10-\mathrm{cm}$ distant from the light source at $45^{\circ} \mathrm{C}$ under air atmosphere.

Commercial PVA powder (degrees of saponification and polymerization, $96 \%$ and 1300 , respectively) and dissolving sulfite pulp from softwoods (degree of polymerization, 750) were used as PVA and cellulose samples, respectively. Photoirradiations were conducted in a quartz glass tube containing $40-\mathrm{m} l$ aqueous solution of PVA or cellulose and $40-\mathrm{m} l$ water. After photoirradiation, in the case of cellulose, the samples were filtered on a glass filter and the filtrate was used for the determination of peroxide.

Carbonyl group was determined according to the hydroxylamine method. ${ }^{3}$ Photo-irradiated aqueous solutions of D-glucose and PVA were allowed to react with a hydroxylamine hydrochloride solution containing bromophenol blue as indicator at $50^{\circ} \mathrm{C}$ for $120 \mathrm{~min}$ and at room temperature for $90 \mathrm{~min}$, and the liberated hydrochloric acid was titrated.

UV spectra of aqueous solutions of saccharide were recorded with a Hitachi spectrophotometer 124 type. A relatively high concentration was chosen for the samples used for UV spectrum measurement $\left(10^{-1} \mathrm{~mol} / \mathrm{l}\right)$, since the usual concentration of saccharide sample $\left(10^{-3}\right.$ to $10^{-5}$ $\mathrm{mol} / l$ ) was too low for observation of changes of saccharide due to irradiation.

\section{RESULTS}

Figure 1 shows the amounts of peroxide formed in various photo-irradiated aqueous solutions of saccharide. The formation of peroxide was observed in the irradiated system using a quartz glass tube under air atmosphere, and increased

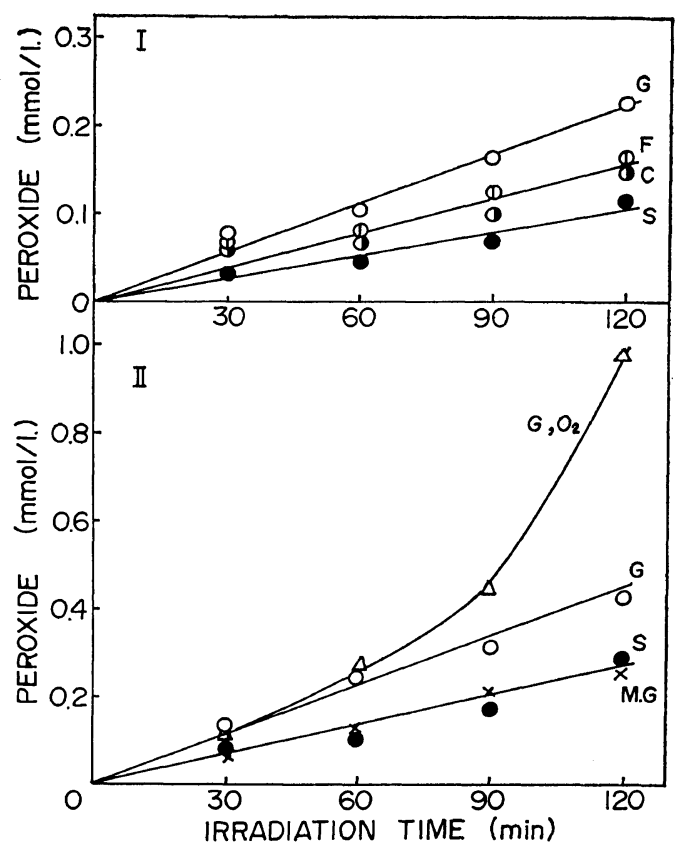

Figure 1. Photo-induced formation of peroxide in aqueous solution of saccharide. Concentration of saccharide: I, $1 \mathrm{mmol} / l$; II, $10 \mathrm{mmol} / l$. G, D-glucose; F, D-fructose; C, cellobiose; $\mathrm{S}$, sucrose; MG, methyl- $\alpha$-D-glucopyranoside; $\mathrm{G}, \mathrm{O}_{2}$, photoirradiation system involving D-glucose(under oxygen atmosphere). Photoirradiations were carried out at $45^{\circ} \mathrm{C}$ in the quartz glass tube under air atmosphere.

linearly with the time of irradiation. A large amount of peroxide was observed after the irradiation under oxygen atmosphere. No formation of peroxide was observed at all in any of the irradiated systems under nitrogen atmosphere, in the absence of saccharide, or using a hard glass tube. Therefore, it is clear that oxygen, saccharide, and light shorter than $300 \mathrm{~nm}$ are three essential factors for the formation of peroxide in this system. A different rate of formation was observed depending upon the kind of saccharide in the order of D-glucose > cellobiose $\approx$ D-fructose $>$ sucrose $\approx$ methyl- $\alpha$-D-glucopyranoside. Rather smaller amounts of peroxide were recorded for systems using nonreducing saccharide compared to those using aldose and ketose. The effect of saccharide concentration on the formation of peroxide was examined in the range from 1 to $50 \mathrm{mmol} / \mathrm{l}$. A higher level of 


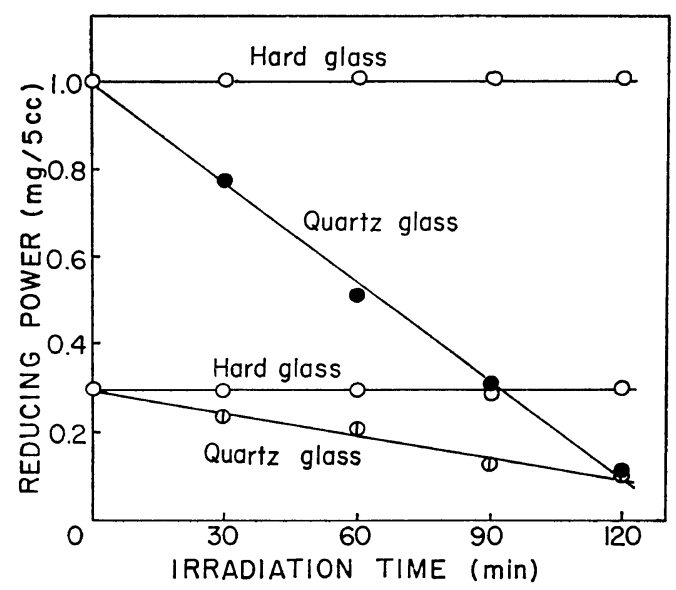

Figure 2. Change in reducing power of D-glucose with photoirradiation at $45^{\circ} \mathrm{C}$. Concentration of D-glucose: $(\bigcirc, \bigcirc), 1.1 \mathrm{mmol} / l ;(\bigcirc,(1), 0.3 \mathrm{mmol} / l$.

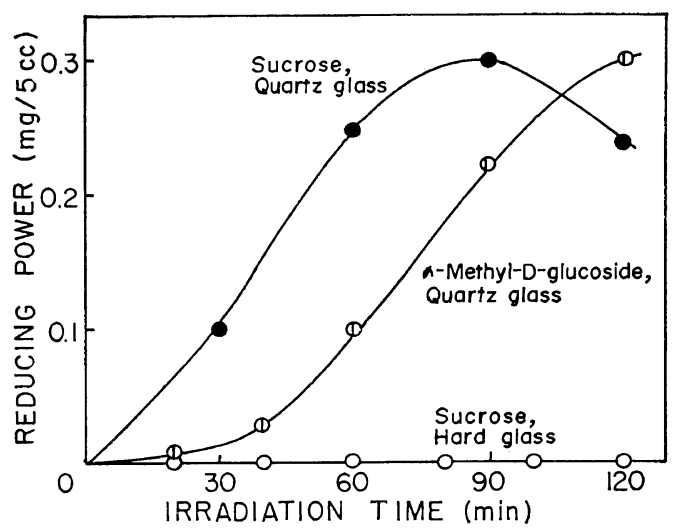

Figure 3. Changes in reducing power of methyl$\alpha$-D-glucopyranoside and sucrose with photoirradiation at $45^{\circ} \mathrm{C}$. Concentration of saccharide, 1.0 $\mathrm{mmol} / l$.

formation was obtained as the concentration of saccharide increased.

Changes of reducing power of D-glucose due to photoirradiation are shown in Figure 2. No change of reducing power observed in the hard glass system, but a marked decrease was shown in the quartz glass system. The same tendency was observed for D-fructose, cellobiose, and maltose. On the other hand, sucrose and methyl- $\alpha$-D-glucopyranoside behaved very peculiarly, and showed an increase in reducing power with photoirradiation (Figure 3). Accordingly, light shorter than $300 \mathrm{~nm}$ clearly contributes to the scission of glycosidic bonds of sucrose and methyl- $\alpha$-D-glucopyranoside, leading to an enhanced reducing power of the system. By analysis of the paper chromatogram ${ }^{4}$ of aqueous solutions of sucrose and methyl- $\alpha$-D-glucopyranoside, a lot of D-glucose was detected in both solutions after irradiation with UV for

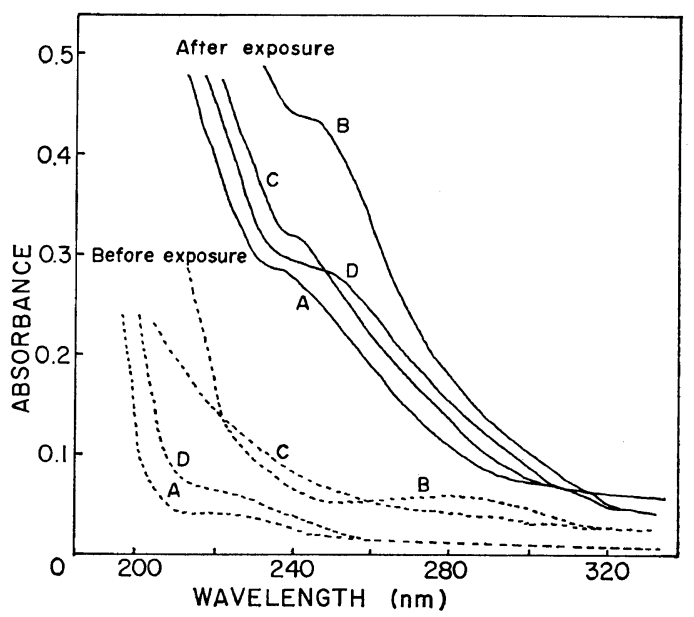

Figure 4. UV spectra of aqueous solutions of saccharide irradiated at $45^{\circ} \mathrm{C}$ for $60 \mathrm{~min}$ in the quartz glass tube under air atmosphere. Concentration of saccharide, $100 \mathrm{mmol} / l$; A, D-glucose; B, Dfructose; C, cellobiose; D, sucrose.

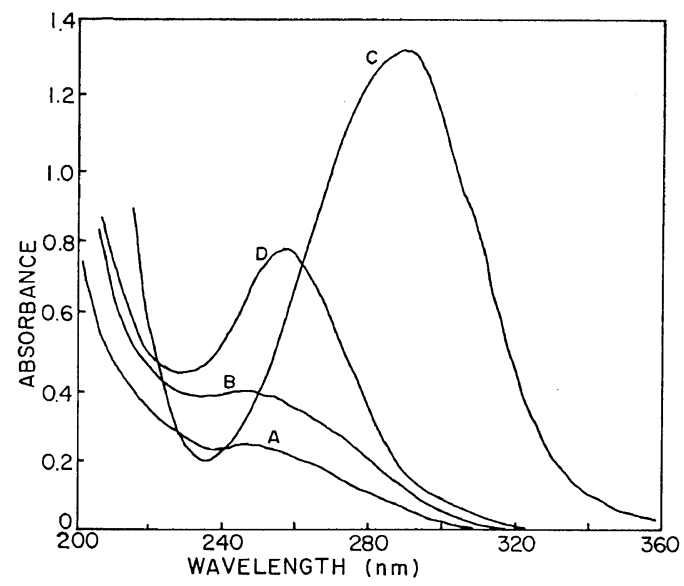

Figure 5. UV spectra of photo-irradiated aqueous solution of D-glucose: A, after 60-min exposure $(\mathrm{pH}=4.2) ; \mathrm{B}$, after hydrochloric acid addition to A $(\mathrm{pH}=2) ; \mathrm{C}$, after sodium hydroxide addition to A $(\mathrm{pH}=12) ; \mathrm{D}$, hydrochloric acid addition to $\mathrm{C}$ $(\mathrm{pH}=5)$. 


\section{H. Kubota, Y. Ogiwara, and K. Matsuzaki}

Table I. UV spectra of irradiated aqueous solutions of saccharide

\begin{tabular}{|c|c|c|c|c|}
\hline \multirow[b]{2}{*}{ Saccharide ${ }^{\mathrm{a}}$} & \multirow{2}{*}{$\begin{array}{l}\mathrm{pH} \text { of } \\
\text { irradiated } \\
\text { solution }\end{array}$} & \multicolumn{3}{|c|}{ Absorption at $\mathrm{nm}$} \\
\hline & & $\begin{array}{l}\text { Irradiated } \\
\text { solution }^{\mathrm{b}}\end{array}$ & $\begin{array}{l}\text { After } \\
\text { addition } \\
\text { of } \mathrm{HCl}\end{array}$ & $\begin{array}{c}\text { After } \\
\text { addition } \\
\text { of } \mathrm{NaOH}\end{array}$ \\
\hline D-Glucose & 4.2 & 245 & 245 & 290 \\
\hline D-Fructose & 4.2 & 242 & 212 & 279 \\
\hline Methyl- $\alpha$-D-glucopyranoside & 4.0 & 245 & 245 & 275 \\
\hline Maltose & 4.1 & 242 & 242 & 272 \\
\hline Cellobiose & 4.3 & 245 & 243 & 290 \\
\hline Sucrose & 4.0 & 248 & 248 & 300 \\
\hline
\end{tabular}

a Concentration of saccharide, $100 \mathrm{mmol} / l$.

b Photoirradiation was carried out at $45^{\circ} \mathrm{C}$ for $60 \mathrm{~min}$ in the quartz glass system under air atmosphere.

$2 \mathrm{hr}$ under air atmosphere. It is obvious that UV light shorter than $300 \mathrm{~nm}$ attacks the saccharide molecule to cleave the glycosidic bonds as well as decompose the reducing end groups.

Figure 4 shows UV spectra of aqueous solution of saccharide irradiated for $60 \mathrm{~min}$ in the quartz glass system under air atmosphere. A new absorption at 242 to $248 \mathrm{~nm}$ was found common to each saccharide after irradiation. The absorption intensity increased with the time of irradiation. The irradiated solution of Dglucose was adjusted to $\mathrm{pH}$ of 2 and 12 using hydrochloric acid and sodium hydroxide, respectively, and the UV absorptions are shown by curves $\mathrm{B}$ and $\mathrm{C}$ in Figure 5, in which peaks are indicated at 245 and $290 \mathrm{~nm}$, respectively. However, UV spectra of unirradiated aqueous solutions of D-glucose were not changed by means of acid or alkali. The absorption peak at 290 $\mathrm{nm}$, which appeared in the solution of $\mathrm{pH} \mathrm{12,} \mathrm{was}$ transferred to $255 \mathrm{~nm}$ and declined in intensity markedly, when the $\mathrm{pH}$ was reduced to 5 using hydrochloric acid (curve D in Figure 5).

Similar examinations were carried out on various irradiated aqueous solutions of saccharide, and the results are summarized in Table I. By adding sodium hydroxide to the irradiated saccharide solution, each spectrum changed into one involving a new absorption at 270 to $300 \mathrm{~nm}$ with large increase in intensity. The $\mathrm{pH}$ of 6.7 of each unirradiated solution of saccharide decreased to 4.0 to 4.3 after irradiation, indicating weak acidity. By adding hydrochloric acid to these solutions, no remarkable change in the wavelength of the peak occurred except
D-fructose, and only a small increase in the intensity took place.

Since the $\mathrm{pH}$ changes of photo-irradiated aqueous solution of saccharide affect the shape of the UV spectrum, there may be a structure capable of tautomerizing between keto and enol isomers. According to the general rule, the keto form is more stable in alkaline medium, which transfers to the enol form as the acidity is enhanced. The changes of spectra are believed to be caused by this isomerism. Laurent ${ }^{5}$ reported generation of a maximum absorption at $267 \mathrm{~nm}$ when an alkaline solution of D-glucose was irradiated with UV light, and one at $245 \mathrm{~nm}$ in an acidic medium. He proposed the structure 4-desoxy5-keto-3,6-mannosaccharolactone. Phillips and Moody observed, in their studies of radiolysis ${ }^{6-8}$ and photolysis ${ }^{9}$ of saccharides, the peak of UV absorption of irradiated samples shifts to longer wavelengths by the addition of alkali.

\section{DISCUSSION}

Either hydroperoxide of saccharide or hydrogen peroxide can be given as a possible type of peroxide produced in photo-irradiated saccharide solution. Phillips and Moody noticed the formation of hydrogen peroxide by means of radiations with UV light ${ }^{9}$ and $\gamma$-ray..$^{6-8,10}$ Moreover, the photooxidation ${ }^{11}$ of isopropyl alcohol by benzophenone is well-known to produce both hydrogen peroxide and acetone according to the following reactions 


$$
\begin{gathered}
\left(\mathrm{C}_{6} \mathrm{H}_{5}\right)_{2} \mathrm{C}=\mathrm{O} \stackrel{{ }^{\nu}}{\longrightarrow}\left(\mathrm{C}_{6} \mathrm{H}_{5}\right)_{2} \mathrm{C}=\mathrm{O}^{*} \\
\stackrel{\mathrm{RH}}{\longrightarrow}\left(\mathrm{C}_{6} \mathrm{H}_{5}\right)_{2} \dot{\mathrm{COH}}+\mathrm{R} . \\
\mathrm{R} \cdot \stackrel{\mathrm{O}_{2}}{\longrightarrow} \mathrm{ROO} \cdot \stackrel{\mathrm{RH}}{\longrightarrow} \mathrm{ROOH}+\mathrm{R} . \\
\mathrm{ROOH} \longrightarrow\left(\mathrm{CH}_{3}\right)_{2} \mathrm{C}=\mathrm{O}+\mathrm{H}_{2} \mathrm{O}_{2}
\end{gathered}
$$

where $\mathrm{RH}$ and $\mathrm{R}$. represent isopropyl alcohol and its radical, $\left(\mathrm{CH}_{3}\right)_{2} \dot{\mathrm{C}} \mathrm{OH}$, respectively.

Hydrogen is abstracted from isopropyl alcohol by attack of excited benzophenone, and the oxyhydroperoxide structure is then formed as an intermediate. The peroxide is supposed to be unstable as it stands, and to decompose easily into acetone and hydrogen peroxide. In an ESR study ${ }^{4}$ of UV-irradiated D-glucose, the authors observed a singlet spectrum which was assigned to a carbon radical with the unpaired electron at the reducing carbon $\mathrm{C}_{1}$. Thus, dehydrogenation at the reducing position takes place easily with the irradiation of light shorter than $300 \mathrm{~nm}$. Therefore, it might be reasonable to consider that the oxyhydroperoxide structure is formed from irradiated saccharide on contact with air. On the other hand, less peroxide was observed for nonreducing saccharide compared to reducing saccharide (Figure 1). Regarding sucrose and methyl- $\alpha$-D-glucopyranoside molecule, the reducing groups originating from photo-cleaved glycosidic bonds may play the chief role in formation of the peroxide (Figure 3). Accordingly, the $C_{1}$ position of $D-$ glucose molecule can be said to be the most appropriate site to form the peroxide. The photolysis mechanism of $\mathrm{D}$-glucose is proposed in Figure 6.

The dissociation energy of a $\mathrm{C}-\mathrm{H}$ bond is known to be generally about $100 \mathrm{kcal} / \mathrm{mol}$, however, light of wavelength $253.7 \mathrm{~nm}$, which is contained in the light source, has sufficient power to break the bond. Beelik and Hamilton ${ }^{12}$ reported that the cellulose molecule has an absorption at about $265 \mathrm{~nm}$ due to the hemiacetal group acting as a weak chromophoric group. Phillips, et al., postulated, with respect to photolyses of D-glucose ${ }^{9}$ and D-sorbitol, ${ }^{13,14}$ that both saccharides decompose directly due to absorption of the light themselves. In the case of D-glucose, the absorbed light energy transfers intramolecularly from the aglycone to the hemiacetal oxygen bridge. In the latter case, nonbonding orbitals on the oxygen atom in D-sorbitol molecule are excited to anti-bonding orbitals $\left(n-\sigma^{*}\right.$ transition), causing the molecule to decompose with light of 230 to $240 \mathrm{~nm}$.

As no formation of peroxide took place in the irradiated system in the absence of saccharide as mentioned previously, the reaction should be related to the direct absorption of light by saccharide. Thus, the dehydrogenation seems to be the essential reaction which is induced by the intramolecular transfer of energy absorbed at the reducing end or by the glycosidic bonds of the saccharide molecule.

According to eq 1 in Figure 6, hydrogen peroxide is given as one product, and the lactone structure as another. As mentioned later, the formation of peroxide was also observed in the system containing PVA. Therefore, it is considered that the position capable of producing peroxide is not limited to the $\mathrm{C}_{1}$ position of the D-glucose molecule, but the $\mathrm{C}_{2}, \mathrm{C}_{3}$, and $\mathrm{C}_{4}$ positions are also available. For example, a mechanism for hydrogen peroxide formation

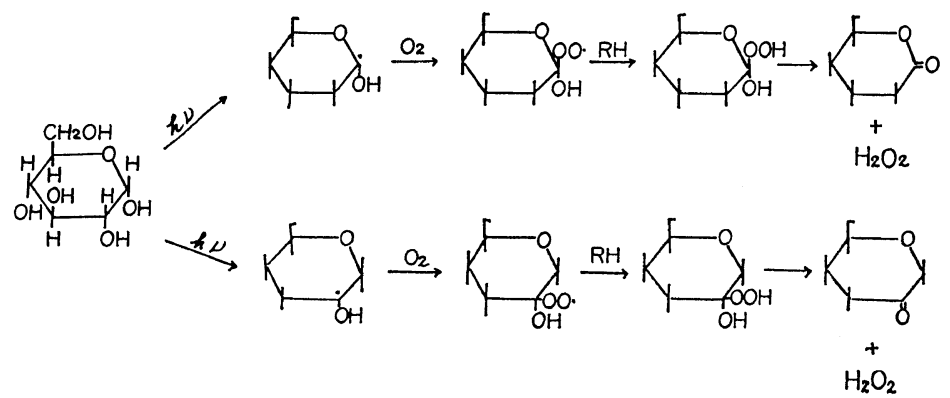

Figure 6. Photo-induced formation of hydrogen peroxide in aqueous solution of D-glucose. RH represents D-glucose molecule. 
Table II. Photo-induced formation of peroxide from cellulose and PVA in aqueous media ${ }^{\mathrm{a}}$

\begin{tabular}{|c|c|c|c|c|c|c|}
\hline \multirow{3}{*}{$\begin{array}{l}\text { Amount of } \\
\text { sample, } \mathrm{g}\end{array}$} & \multirow{2}{*}{\multicolumn{2}{|c|}{$\begin{array}{c}\text { Concn of sample } \\
\text { unit } \mathrm{mol} / l^{\mathrm{b}}\end{array}$}} & \multicolumn{4}{|c|}{ Peroxide, $\mathrm{mmol} / l$} \\
\hline & & & \multicolumn{2}{|c|}{ Cellulose } & \multicolumn{2}{|c|}{ PVA } \\
\hline & Cellulose & PVA & $1 \mathrm{hr}$ & $2 \mathrm{hr}$ & $1 \mathrm{hr}$ & $2 \mathrm{hr}$ \\
\hline 0.1 & 1.54 & 5.68 & 0.04 & 0.11 & 0.32 & 0.35 \\
\hline 0.5 & 7.70 & 28.4 & 0.22 & 0.35 & 0.23 & 0.30 \\
\hline 1.0 & 15.4 & 56.8 & 0.26 & 0.30 & 0.20 & 0.26 \\
\hline
\end{tabular}

a Photoirradiation was carried out at $45^{\circ} \mathrm{C}$ in the quartz glass system under air atmosphere.

b Unit $\mathrm{mol} / \mathrm{l}$ is the concentration calculated from the molecular weights of each repeating unit of cellulose and $\mathrm{PVA},-\mathrm{C}_{6} \mathrm{H}_{10} \mathrm{O}_{5}-$ and $-\mathrm{CH}_{2} \mathrm{CH}(\mathrm{OH})$-, respectively.

involving the $\mathrm{C}_{2}$ position is proposed in eq 2 in Figure 6 in which the D-glucose molecule is transformed into a structure containing a ketone group.

The final compounds shown in eq 1 and 2 will certainly exhibit keto-enol tautomerism adopting stable keto and enol forms in alkaline and acidic media, respectively. In the qualitative test for lactone by the hydroxamic acid method, ${ }^{15}$ with both $\mathrm{D}$-glucose and cellobiose, the red-brown color clearly increased after irradiation of $2 \mathrm{hr}$ in the quartz glass system under air atmosphere, proving the formation of lactone structures. This supports the reaction scheme in eq 1 in Figure 6.

Changes in the amount of carbonyl group in aqueous solutions of D-glucose with UV-irradiation were examined. The amount of carbonyl group was observed to increase with the time of irradiation. For example, the amount of increase was $2.8 \mathrm{mmol} / l$ for the system involving $50 \mathrm{mmol} / l \mathrm{D}$-glucose at 4-hr irradiation, and 11.5 $\mathrm{mmol} / l$ increase for the system of $100 \mathrm{mmol} / l$ D-glucose. On the other hand, an appreciable amount of decomposed reducing group of Dglucose was determined under the same UVirradiation conditions shown in Figure 2. Therefore, the fact that UV-irradiation increased the amount of carbonyl group in the aqueous solution of D-glucose suggests the formation of fresh carbonyl groups in the system, which perhaps are nonreducing in character. Thus, the final compound could be considered to include a ketone structure of the type shown in eq 2 .

Amounts of peroxide in irradiated PVA and cellulose samples are shown in Table II. The value was maximum at certain amounts of
PVA and cellulose. The reducing end group of the cellulose molecule is inferred to contribute effectively to the formation of peroxide as in the case of saccharides. Glycosidic bonds of the cellulose molecule might be photolyzed ${ }^{16}$ to produce fresh reducing end groups capable of effectively initiating the formation of peroxide. In the PVA sample, the reaction may be initiated by light through the abstraction of a hydrogen atom from the $\alpha$-carbon bonded to the hydroxyl group, leading to the formation of a ketone structure on the main chain. Determination of carbonyl group in the photo-irradiated aqueous solutions of PVA confirmed its formation in the system and the amount increased linearly with the time of irradiation. This result seems to support the introduction of a ketone structure into the PVA molecule. By studying the ESR of UV-irradiated cellulose ${ }^{17}$ and $\mathrm{PVA}^{18},{ }^{18}$ the authors observed dehydrogenation from the main chain with light shorter than $300 \mathrm{~nm}$.

From the above examinations, it is clear that formation of hydrogen peroxide by photolysis occurs in saccharide and cellulose preferentially at the reducing groups. On the other hand, PVA can also form peroxide in the same way by involvement of the $-\mathrm{HC}(\mathrm{OH})$ - structure. Therefore, it is concluded that the structure $-\mathrm{HC}(\mathrm{OH})$ - contributes in a wide sense to the formation of peroxide in saccharide and related compounds as the primary reaction site during photolysis.

\section{REFERENCES}

1. H. Kubota, Y. Ogiwara, and K. Matsuzaki, J. Appl. Polym. Sci., 20, 1405 (1976). 
2. G. O. Phillips, Advan. Carbohyd. Chem., 18, 9 (1963).

3. "Synthetic Method of Monomers," Kobunshi Jikkengaku Koza, Vol. 9, The Society of Polymer Science, Japan, Ed., Kyoritsu Publishing Co., Ltd., Tokyo, 1957, p 188.

4. H. Kubota, Y. Ogiwara, and K. Matsuzaki, J. Appl. Polym. Sci., 19, 1291 (1975).

5. T. C. Laurent, J. Amer. Chem. Soc., 78, 1875 (1956).

6. G. O. Phillips, G. J. Moody, and G. L. Mattok, J. Chem. Soc., 3522 (1968).

7. G. O. Phillips and G. J. Moody, ibid., 754 (1959).

8. G. O. Phillips and G. J. Moody, ibid., 762 (1960).

9. G. O. Phillips and G. J. Moody, ibid., 3398 (1960).

10. G. O. Phillips and K. W. Davies, ibid., 205
(1964).

11. K. Tokumaru, "Yuki Kokagaku Hannoron," Tokyo Kagaku Dojin, Tokyo, 1973, p 6.

12. A. Beelik and J. K. Hamilton, Das Papier, 13, 77 (1959); J. Org. Chem., 26, 5074 (1961).

13. G. O. Phillips and W. J. Criddle, J. Chem. Soc. 3984 (1963).

14. G. O. Phillips and P. Barber, ibid., 3990 (1963).

15. "Qualitative and Confirming Method of Organic Compounds," Jikken Kagaku Koza, Vol. 5, The Chemical Society, Japan, Ed., Maruzen, Tokyo, 1966, p 690.

16. Y. Ogiwara, H. Kubota, and Y. Murata, $J$. Appl. Polym. Sci., 18, 3455 (1974).

17. Y. Ogiwara and H. Kubota, J. Polym. Sci., Polym. Chem. Ed., 11, 3243 (1973).

18. Y. Ogiwara, T. Yasunaga, and H. Kubota, $J$. Appl. Polym. Sci., 19, 1119 (1975). 